\title{
Sub-microsecond entangling gate between trapped ions via Rydberg interaction
}

\author{
Chi Zhang, ${ }^{1, *}$ Fabian Pokorny, ${ }^{1}$ Weibin Li, ${ }^{2,3}$ Gerard Higgins, ${ }^{1}$ \\ Andreas Pöschl, ${ }^{1}$ Igor Lesanovsky, ${ }^{2,3,4}$ and Markus Hennrich ${ }^{1, \dagger}$ \\ ${ }^{1}$ Department of Physics, Stockholm University, 10691 Stockholm, Sweden \\ ${ }^{2}$ School of Physics and Astronomy, University of Nottingham, \\ Nottingham, NG7 2RD, United Kingdom \\ ${ }^{3}$ Centre for the Mathematics and Theoretical Physics of Quantum Non-equilibrium Systems, \\ University of Nottingham, Nottingham, NG7 2RD, United Kingdom \\ ${ }^{4}$ Institut für Theoretische Physik, Universität Tübingen, \\ Auf der Morgenstelle 14, 72076 Tübingen, Germany
}




\begin{abstract}
Generating quantum entanglement in large systems on time scales much shorter than the coherence time is key to powerful quantum simulation and computation. Trapped ions are among the most accurately controlled and best isolated quantum systems [1] with low-error entanglement gates operated within tens of microseconds using the vibrational motion of few-ion crystals $[2,3]$. To exceed the level of complexity tractable by classical computers the main challenge is to realise fast entanglement operations in large ion crystals [4]. The strong dipole-dipole interactions in polar molecule [5] and Rydberg atom [6,7] systems allow much faster entangling gates, yet stable state-independent confinement comparable with trapped ions needs to be demonstrated in these systems [8]. Here, we combine the benefits of these approaches: we report a $700 \mathrm{~ns}$ two-ion entangling gate which utilises the strong dipolar interaction between trapped Rydberg ions and produce a Bell state with $78 \%$ fidelity. The sources of gate error are identified and a total error below $0.2 \%$ is predicted for experimentally-achievable parameters. Furthermore, we predict that residual coupling to motional modes contributes $\sim 10^{-4}$ gate error in a large ion crystal of 100 ions. This provides a new avenue to significantly speed up and scale up trapped ion quantum computers and simulators.
\end{abstract}

Trapped atomic ions are one of the most promising architectures for realizing a universal quantum computer [1]. The fundamental single- and two-qubit quantum gates have been demonstrated with errors less than $0.1 \%[2,3]$, sufficiently low for fault-tolerant quantum error-correction schemes. Single-qubit coherence time of $10 \mathrm{~min}$ [9] as well as the proofof-principle demonstration of error correction $[10,11]$ have been realised. Nevertheless, a scalable quantum computer requires a large number of qubits and a large number of gate operations to be conducted within the coherence time. Most established gate schemes using a common motional mode are slow (typical gate times are between 40 and $100 \mu \mathrm{s}$ ) and difficult to scale up since the motional spectrum becomes more dense with increasing ion number. Many new schemes have been proposed and implemented [12, 13], with the fastest experimentally-achieved gate in a two-ion crystal being $1.6 \mu \mathrm{s}$ (99.8\% fidelity) and $480 \mathrm{~ns}$ (60\% fidelity) [13], realised by driving multiple motional modes simultaneously. Although the gate speed is not limited by the trap frequencies, the gate protocol requires the phase-

\footnotetext{
* chi.zhang@fysik.su.se

† markus.hennrich@fysik.su.se
} 
space trajectories of all modes to close simultaneously at the end of the pulse sequence [13]. In long ion strings with a large number of vibrational modes, it becomes increasingly challenging to find and implement laser pulse parameters that execute this gate with a low error. Thus, a slow-down of gate speed appears inevitable.

Two-qubit entangling gates in Rydberg atom systems [7] are substantially faster, owing to strong dipole-dipole interactions. The gate fidelities in recent experiments using neutral atoms are fairly high $[14,15]$. However, the atom traps need to be turned off during Rydberg excitation. This can cause unwanted coupling between qubits and atom motion as well as atom loss [8]. Employing blue-detuned optical tweezers at a magic wavelength one may achieve the trapping of Rydberg states, though the predicted residual change in trapping frequency of $\sim 50 \%$ [16] will still result in entanglement between qubits and motional states and thus a reduction in gate fidelity. In addition, switching between different trapping potentials or switching off and on the trap will exponentially heat up the atoms [17] and reduce the gate fidelity (the unwanted motional effects are stronger at higher temperatures). Since cooling without destroying the qubit information is challenging (direct cooling by lasers will destroy the qubit, while sympathetic cooling will cause entanglement between qubit and coolant atoms unless their interaction is state-independent [18]), this may limit the number of gate operations despite the long coherence times of these systems.

In solid-state platforms, like superconducting circuits [19] and silicon-based qubits [20], the interactions are also strong, enabling fast two-qubit gates, and tremendous progress has been made recently. However, the number of entanglement operations that can be executed in the coherence time using these systems is typically $\sim 10^{3}$ (with superconducting circuits gate times $\sim 50 \mathrm{~ns}$ and coherence times $\sim 100 \mu \mathrm{s}$ ), which is orders of magnitude less than $\sim 10^{6}$ in atomic systems (with trapped ions gate times $\sim 100 \mu$ s and coherence times $\sim 100 \mathrm{~s}$, Rydberg gates may improve this to $\sim 10^{8}$ ).

Combining the benefits of trapped ion qubits and Rydberg interactions is a promising approach for scalable quantum computation [21]: Rydberg interactions may enable fast, motion-independent gates between trapped ions. Additionally, because ions are trapped via their electric charges and interact via the state-independent Coulomb interaction, Rydberg ions do not suffer from most of the limitations of neutral Rydberg atom systems. In previous works it was shown that ions in Rydberg states can be confined [22, 23] and coherence between Rydberg and low-lying states can be maintained [24] in radio-frequency traps. 
However, strong interactions between Rydberg ions and their use for fast entangling gates had not been previously demonstrated.

In our experiment ${ }^{88} \mathrm{Sr}^{+}$ions are confined in a linear Paul trap. Two low-lying electronic states $(|0\rangle$ and $|1\rangle)$ are used to store a qubit, and $|0\rangle$ is coupled to Rydberg state $|r\rangle$ via a two-photon laser field. The relevant level scheme is shown in Fig. 1(a), more details can be found in [24].

Two ions excited to Rydberg states interact through the dipole-dipole interaction

$$
\hat{V}_{\mathrm{dd}}=\frac{1}{4 \pi \epsilon_{0}}\left(\frac{\hat{\boldsymbol{\mu}}_{\mathbf{1}} \cdot \hat{\boldsymbol{\mu}}_{\mathbf{2}}-3\left(\hat{\boldsymbol{\mu}}_{\mathbf{1}} \cdot \mathbf{n}\right)\left(\hat{\boldsymbol{\mu}}_{\mathbf{2}} \cdot \mathbf{n}\right)}{|\mathbf{r}|^{3}}\right)
$$

where $\hat{\boldsymbol{\mu}}_{\boldsymbol{i}}$ is the electric dipole moment of ion $i(i=1,2), \mathbf{r}=\mathbf{r}_{\mathbf{2}}-\mathbf{r}_{\mathbf{1}}$ is the relative ion position and $\mathbf{n}=\mathbf{r} /|\mathbf{r}|$. Trapped ions in atomic eigenstates have zero dipole moments and $\hat{V}_{\text {dd }}$ has no first-order effect. The second-order effect (van der Waals interaction) can be sufficiently strong to cause Rydberg blockade in neutral atom systems with principal quantum number $n \sim 50$ within a few $\mu \mathrm{m}$ [25]. However, this interaction is much weaker in Rydberg ion systems; it scales with net core charge as $Z^{-6}$ (for $\mathrm{Sr}^{+}$with one valence electron $Z=2$ ). Instead, we achieve a strong first-order interaction by inducing rotating electric dipole moments via a microwave (MW) field.

When two Rydberg states $|s\rangle$ and $|p\rangle$ are coupled by a MW field with Rabi frequency $\Omega_{\mathrm{MW}}$ and detuning $\Delta_{\mathrm{MW}}\left[\right.$ Fig. 1 (b)], the eigenstates become $| \pm\rangle=C\left(\frac{\Delta_{\mathrm{MW}} \pm \sqrt{\Delta_{\mathrm{MW}}^{2}+\Omega_{\mathrm{MW}}^{2}}}{\Omega_{\mathrm{MW}}}|s\rangle+|p\rangle\right)$, where $C$ is the normalization constant [26]. In our system the electric dipole moments of the dressed states $| \pm\rangle$ rotate with the MW field in the plane perpendicular to the magnetic field, as shown in Fig. 1(c) and (d). For two ions, each in state $|r\rangle \equiv|+\rangle$, the dipole-dipole interaction given by Eq. (1) yields an energy shift

$$
V\left(\Delta_{\mathrm{MW}}, \Omega_{\mathrm{MW}}\right)=\left\langle r r\left|\hat{V}_{\mathrm{dd}}\right| r r\right\rangle=\frac{1}{4 \pi \epsilon_{0}} \frac{\langle s|\hat{\mu}| p\rangle^{2}}{r^{3}}\left(\frac{\Omega_{\mathrm{MW}}^{2}}{\Delta_{\mathrm{MW}}^{2}+\Omega_{\mathrm{MW}}^{2}}\right) \propto \frac{n^{4}}{Z^{2}}
$$

with maximum interaction strength $V_{\max }=\frac{1}{4 \pi \epsilon_{0}} \frac{\langle s|\hat{\mu}| p\rangle^{2}}{r^{3}}$. For the measurements described in this letter, we use Rydberg states with principal quantum number $n=46$ and ion separation $4.2 \mu \mathrm{m}$, resulting in $V_{\max } \simeq 2 \pi \times 1.9 \mathrm{MHz}$. By tuning the ratio between $\Omega_{\mathrm{MW}}$ and $\Delta_{\mathrm{MW}}$ the interaction strength can be varied between $\sim 0$ and $V_{\max }$. Higher-order terms in $\hat{V}_{\mathrm{dd}}$ can be neglected since the energy splitting between dressed states $\Omega_{\mathrm{MW}} \gg V_{\max }$.

We probe the interaction between Rydberg ions as shown in the Rabi oscillations between $|0\rangle$ and $|r\rangle$ in Fig. 2. Either one or two ions are trapped and initialised in $|0\rangle$. A two-photon 


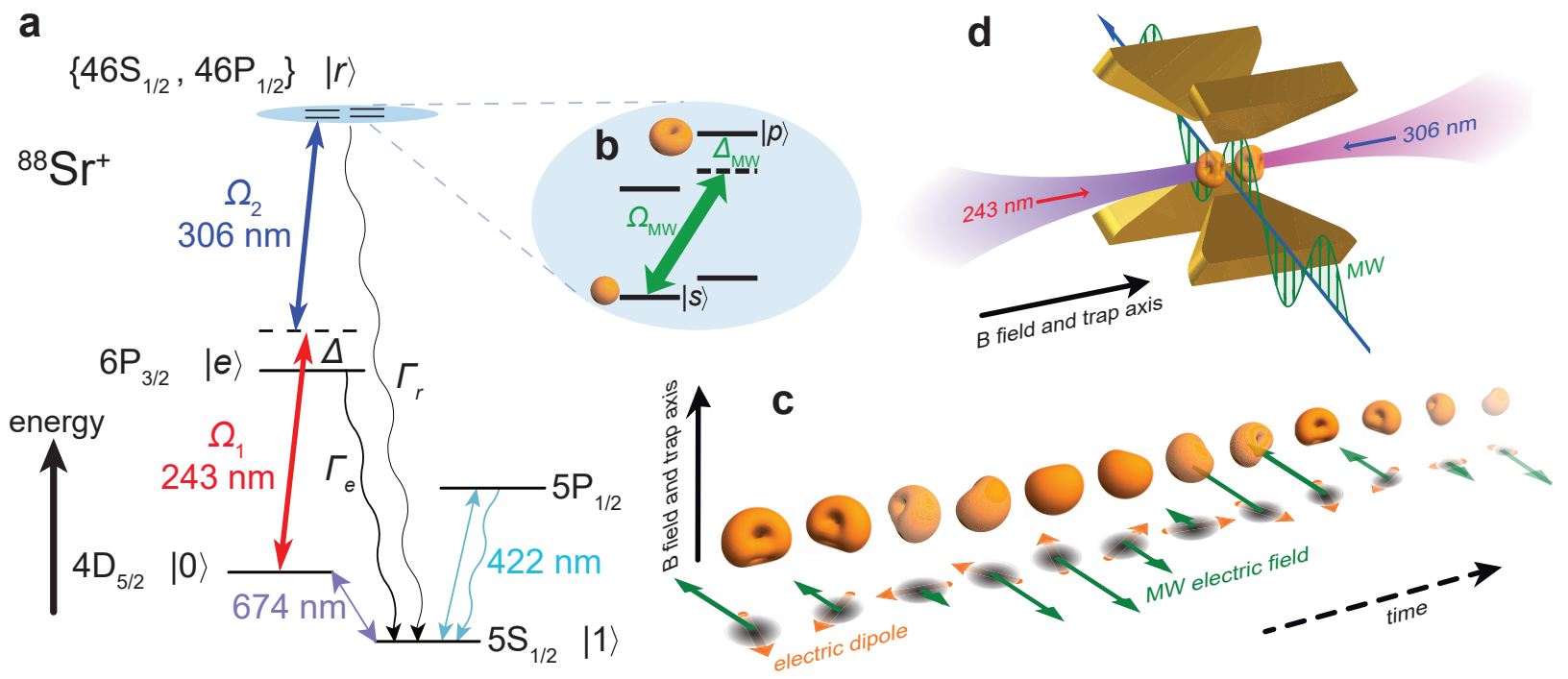

FIG. 1. Level scheme of ${ }^{88} \mathrm{Sr}^{+}$and the rotating dipole moment of a MW-dressed Rydberg state. (a) Ground state $5 S_{1 / 2}, m_{J}=-1 / 2$ and metastable state $4 D_{5 / 2}, m_{J}=-5 / 2$ are used to store a qubit $\{|1\rangle,|0\rangle\}$. The qubit transition is driven by a $674 \mathrm{~nm}$ laser field. Projective measurements in any basis are carried out by qubit rotations and fluorescence detection at $422 \mathrm{~nm}$. Rydberg excitation is driven by a two-photon laser field; $243 \mathrm{~nm}$ laser field couples $|0\rangle \leftrightarrow|e\rangle\left(6 P_{3 / 2}, m_{J}=\right.$ $-3 / 2)$ and $306 \mathrm{~nm}$ laser field couples $|e\rangle \leftrightarrow|s\rangle\left(46 S_{1 / 2}, m_{J}=-1 / 2\right)$. (b) A continuous wave MW field at $122 \mathrm{GHz}$ couples Rydberg state $|s\rangle$ with $|p\rangle\left(46 P_{1 / 2}, m_{J}=1 / 2\right)$. (c) The Rydberg electron density for the MW-dressed state $\frac{1}{\sqrt{2}}(|s\rangle+|p\rangle)$ yields a permanent dipole which rotates in antiphase with the MW electric field about the magnetic field direction. (d) The two MW-dressed Rydberg ions interact via the dipole-dipole interaction. They are confined on the trap axis.

laser field then couples $|0\rangle \leftrightarrow|r\rangle$, the pulse length is varied and population that is excited to $|r\rangle$ and subsequently decays to $5 S_{1 / 2}$ is measured by fluorescence detection (more details about the detection scheme is described in our previous work [24]). From $(b) \rightarrow(d)$ the dipoledipole interaction strength is increased: this has no effect on the Rabi oscillations when a single ion is trapped (green data), while two-ion oscillations (red data) are suppressed as the interaction shifts the pair state $|r r\rangle$ out of resonance from the laser excitation. When $|r r\rangle$ is far from resonance the population oscillates between two states: $|00\rangle$ and the Bell state $\frac{1}{\sqrt{2}}(|0 r\rangle+|r 0\rangle)$. This is the blockade regime [27, 28], which is corroborated by the $\sqrt{2}$ enhancement of two-ion Rabi oscillation frequency (blue data) over the single-ion oscillation frequency (green data) in (d). 


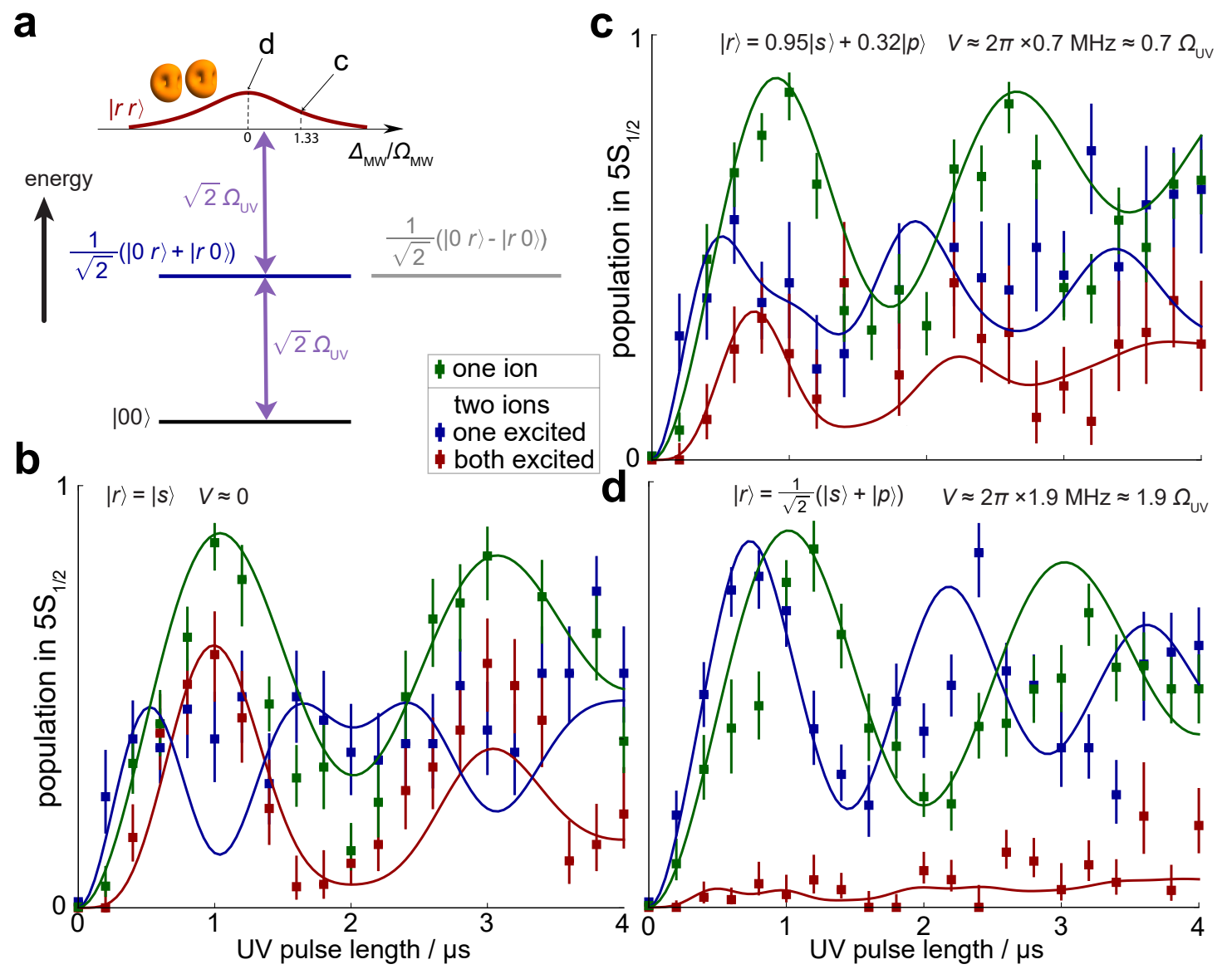

FIG. 2. Tunable interaction between Rydberg ions. (a) Two-ion level scheme, where levels are coupled by the two-photon laser field $\Omega_{\mathrm{UV}}=\frac{\Omega_{1} \Omega_{2}}{2 \Delta}$, and the energy of state $|r r\rangle$ is shifted by the tunable Rydberg interaction. (b)-(d) Rabi oscillations between $|0\rangle \leftrightarrow|r\rangle$ for one and two ions. From $(\mathrm{b}) \rightarrow(\mathrm{d})$ the interaction strength $V$ is increased from 0 to $V_{\max }$ by tuning the MW field amplitude and detuning. The y-axes show the population which is excited to $|r\rangle$ and subsequently decays to $5 S_{1 / 2}$. Green data points for one-ion excitation $|0\rangle \leftrightarrow|r\rangle$ are similar in (b)-(d). Red data points show the two-ion excitation probability for $|00\rangle \leftrightarrow|r r\rangle$ which, in (b), is similar to the square of the one-ion (green) data. From $(\mathrm{b}) \rightarrow(\mathrm{d})$ excitation to $|r r\rangle$ is increasingly suppressed as $|r r\rangle$ is shifted out of resonance from the laser excitation, with Rydberg blockade in (d). The blue data points show the two-ion excitation probability for $|00\rangle \leftrightarrow \frac{1}{\sqrt{2}}(|0 r\rangle+|r 0\rangle)$; a $\sqrt{2}$-enhanced Rabi frequency compared with the one-ion case (green) is observed in the blockade regime (d). Error bars indicate one standard deviation due to quantum projection noise. Dephasing is mainly caused by finite Rydberg lifetime and laser linewidth. Solid lines show results of numerical simulations of the Lindblad Master equation including all known decoherence sources and using no free parameters. More details of the numerical simulation can be found in the Supplemental Information. 


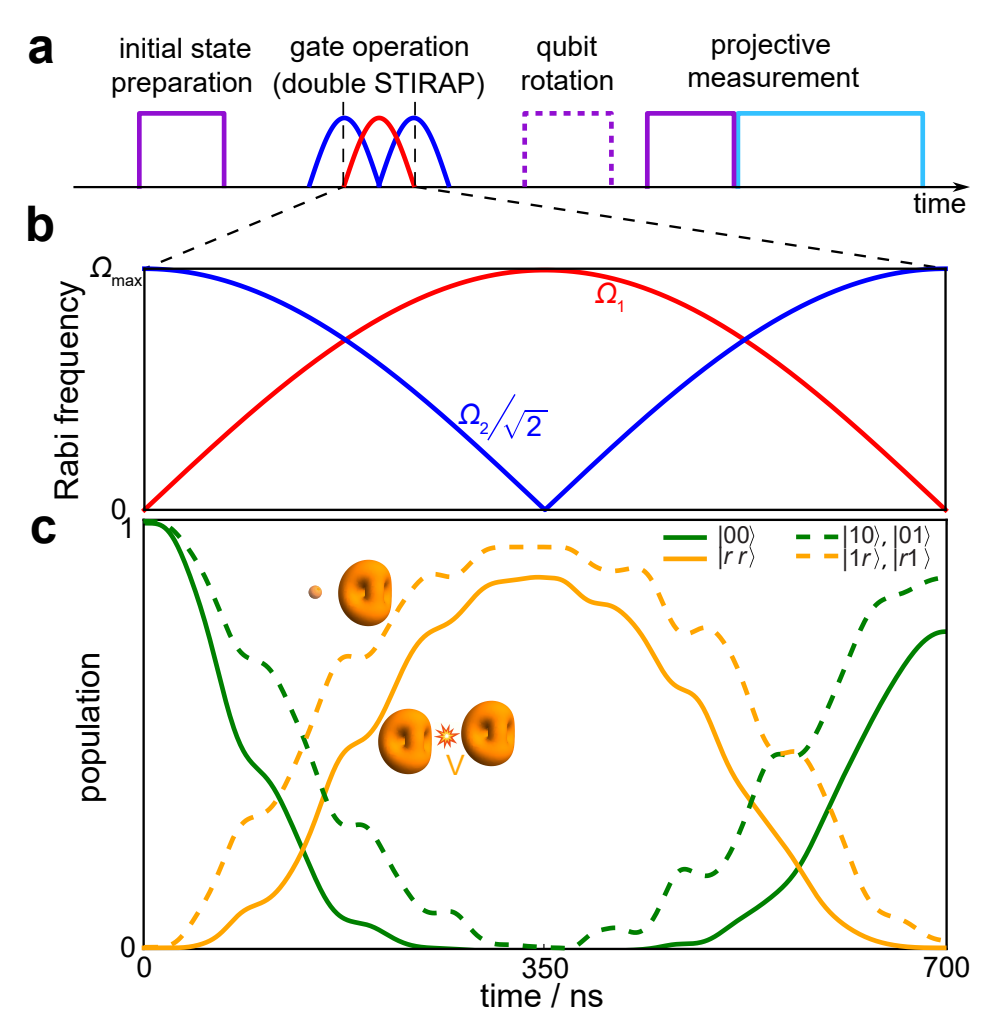

FIG. 3. Experimental sequence of the Rydberg-interaction gate. (a) Pulse sequence: two ions are prepared in $\frac{1}{2}(|0\rangle+|1\rangle)(|0\rangle+|1\rangle)$, followed by the gate operation involving Rydberg excitation using a double-STIRAP sequence, the qubit rotation is applied for parity-oscillation measurements, and finally the two-ion state is measured using qubit rotation and fluorescence detection. (b) Gate operation: the $|0\rangle \leftrightarrow|e\rangle$ and $|e\rangle \leftrightarrow|r\rangle$ coupling strengths $\left(\Omega_{1}\right.$ and $\left.\Omega_{2} / \sqrt{2}\right)$ are varied sinusoidally over 700 ns. (c) Simulation of one- and two-ion population dynamics during gate operation, the controlled-phase accumulated by $|00\rangle$ is proportional to area enclosed by the $|r r\rangle$ population curve. Other states $(|01\rangle,|10\rangle$ and $|11\rangle)$ do not accumulate phases since their eigenenergies remain zero.

We then use the maximum interaction strength $\left(V=V_{\max }\right)$ to implement a $700 \mathrm{~ns}$ controlled phase gate between two ions. The experimental sequence is described in Fig. 3. First the two ions are initialized in state $\frac{1}{2}(|00\rangle+|01\rangle+|10\rangle+|11\rangle)$, then the gate operation is applied - population is transferred from $|0\rangle \rightarrow|r\rangle \rightarrow|0\rangle$, and the Rydberg interaction causes component $|00\rangle$ to acquire phase $\phi$. Finally qubit rotations and projective measurements are used to determine the final two-ion state.

The gate operation consists of a double stimulated Raman adiabatic passage (STIRAP) pulse sequence $[24,29]$. The three levels $|0\rangle,|e\rangle$ and $|r\rangle$ are coupled by two laser fields with 

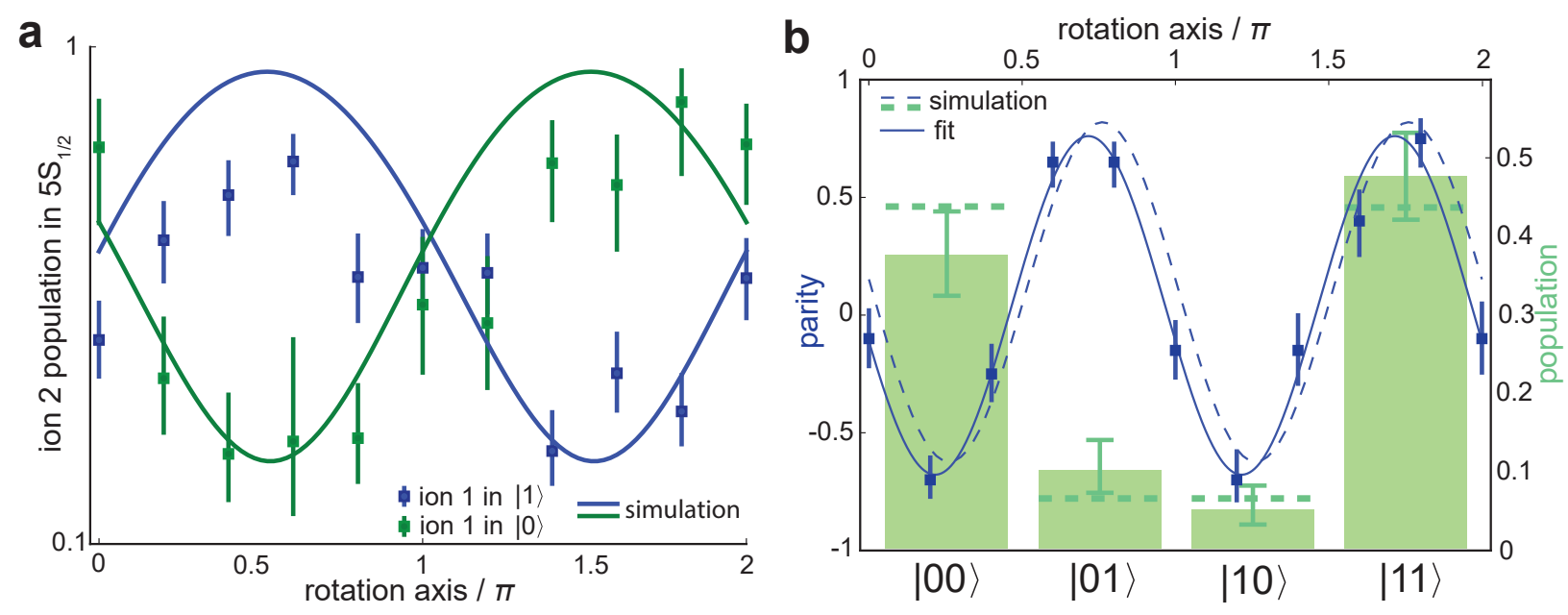

FIG. 4. Analysis of the two-ion state after the entangling gate operation. (a) A Ramsey-type experiment measures the relative phase between $|0\rangle_{2}$ and $|1\rangle_{2}\left(\frac{\pi}{2}\right.$-pulse of varied phase applied on ion 2 followed by projection measurement on $\left.\left\{|0\rangle_{2},|1\rangle_{2}\right\}\right)$ conditional on the state of ion 1 ; the $\pi$-phase difference between the cases $\left\{|0\rangle_{1},|1\rangle_{1}\right\}$ is consistent with the target entangled state $\frac{1}{2}\left(|0\rangle_{1}\left(-|0\rangle_{2}+|1\rangle_{2}\right)+|1\rangle_{1}\left(|0\rangle_{2}+|1\rangle_{2}\right)\right)$. The mismatch between simulation and data is mainly because of imperfect single ion addressing of the qubit laser beam. (b) (78 \pm 3$) \%$ entanglement fidelity is determined by population (green) and parity-oscillation (blue) measurements after rotating the target state to the Bell state $\frac{1}{\sqrt{2}}(|00\rangle+i|11\rangle)$. The small phase mismatch between simulation and experiment (due to minor dynamical phase) does not affect the entanglement fidelity. Error bars in both (a) and (b) indicate one standard deviation due to quantum projection noise.

coupling strengths $\Omega_{1}$ and $\Omega_{2} .|0\rangle$ and $|r\rangle$ are resonantly coupled while $|e\rangle$ is detuned by $\Delta$ (see Fig. 1). $\Omega_{1}$ and $\Omega_{2}$ are gradually changed such that an ion initially in $|0\rangle$ adiabatically follows an eigenstate to $|r\rangle$ and back to $|0\rangle$. An ion initially in $|1\rangle$ is unaffected. For the initial pair states $|11\rangle,|10\rangle$ and $|01\rangle$ the eigenenergies remain zero and no phase is accumulated. From initial state $|00\rangle$ population can be excited to $|r r\rangle$ (provided $\Delta \gtrsim V_{\max }$, more details can be found in the Supplemental Information or reference [29]), the energy of which is shifted due to the Rydberg interaction. Thus $|00\rangle$ acquires the phase $\phi=V_{\max } \int_{0}^{T}\langle r r|\rho(t)| r r\rangle d t$, with the two-ion density operator $\rho(t)$ and pulse length $T$. We achieve $\phi \simeq \pi$ using sinusoidal profiles for $\Omega_{1}(t), \Omega_{2}(t)$ and $T=\frac{8 \pi}{3 V_{\max }} \simeq 700 \mathrm{~ns}$. In the ideal case the final target state $\frac{1}{2}(-|00\rangle+|01\rangle+|10\rangle+|11\rangle)$ is maximally entangled.

The correlation of the final state is measured as follows: the projection of ion 1 on 
$\left\{|0\rangle_{1},|1\rangle_{1}\right\}$ and the phase between $|0\rangle_{2}$ and $|1\rangle_{2}$ of ion 2 are measured simultaneously, results are shown in Fig. 4(a). The phase between $|0\rangle_{2}$ and $|1\rangle_{2}$ of ion 2 is $0(\pi)$ when ion 1 is projected onto $|1\rangle_{1}\left(|0\rangle_{1}\right)$ - this indicates $\phi=\pi$. Entanglement is characterized by parityoscillation measurements [30] after rotating the target state to a Bell state [Fig. 4(b)]. The coherence and population of the Bell state are measured to be $C=0.72 \pm 0.04$ and $P=P_{|00\rangle}+P_{|11\rangle}=0.85 \pm 0.04$, which give an entanglement fidelity of $F=\frac{P+C}{2}=0.78 \pm 0.03$. The uncertainty of the fidelity is determined by Monte Carlo simulations described in the Supplemental Information.

The contributions of different gate error sources are estimated by numerical simulation (Table I). They can account for the observed gate infidelity. The largest error contributions are technical and can be diminished by improving the MW power stability, increasing the laser intensities and decreasing the laser linewidths. Further, several error contributions depend on the gate time, which can be reduced by using higher Rydberg states which interact more strongly, together with higher laser intensities. In our gate implementation we use sideband cooling to mitigate mechanical effects of Rydberg ion polarisability [23, 31], which will become unnecessary when we use MW-dressed Rydberg states with zero polarisability [26]. In turn, this may allow implementation of the gate in higher-dimensional ion crystals. Importantly, we observe no heating effects of the ion motion after the gate operation in the two-ion crystal. Numerical simulation indicates the gate error induced by mechanical forces between interacting Rydberg ions, which can excite ion motion, is $\sim 10^{-4}$ in a 100 -ion crystal using a zero-polarisability MW-dressed state. This simulation and more details about gate errors due to mechanical effects in large ion crystals can be found in the Supplemental Information.

In summary, we have demonstrated a strong Rydberg dipole-dipole interaction $(2 \pi \times$ $1.9 \mathrm{MHz}$ ) and a $700 \mathrm{~ns}$ controlled phase gate in a trapped ion experiment. This provides a new and promising way to increase both the number of entangling operations within the coherence time and the number of qubits in a trapped ion quantum computer or simulator. Although gates based on Rydberg interactions have already been demonstrated for neutral atoms, their availability in trapped ion systems offers a host of new opportunities and advantages.

Ions are trapped by their electric charges, this enables deep, tight and state-independent confinement. The spread of the motional wavefunction is typically $\sim 10 \mathrm{~nm}$, much smaller 
than the effective excitation laser wavelengths, as a result the system is in the Lamb-Dicke regime and motion is largely decoupled from electronic transitions.

The state-independent Coulomb interaction enables many important techniques in trapped ion systems, for example, sympathetic cooling [32] and multi-element logic gates [33], which are compatible with the Rydberg gate. The ion crystal can be cooled via the common motional modes without affecting the qubits or the Rydberg gate operation (since the gate does not rely on the motional modes), thus it is protected from heating by the trap or other noise sources. The advantages of different ion species can be combined, for example, qubit information can be transferred to ions with long coherence times for storage and to ions with strong interactions for gate operations.

The insensitivity of the Rydberg gate to ion temperature and motional modes enables its application in scalable architectures, such as large or even higher-dimensional ion crystals and ion shuttling systems [34]. A Rydberg gate may be applied using a fixed pulse sequence to achieve a robust gate fidelity in large crystals or shuttling systems despite the difficulty in reaching low ion temperatures, and even though the number of ions and the motional modes vary. Currently we explored a short-range Rydberg interaction, but inside a MW cavity the interaction can be made all-to-all without decreasing with distance.

Furthermore, the combination of the Rydberg dipole-dipole interaction and the Coulomb interaction may enable coupling between electronic and vibrational degrees of freedom, and thus interesting quantum simulations, for example, tunable multi-body interactions [35] or excitation transport through motion in biomolecules [36].

[1] Wineland, D. J. et al. Experimental issues in coherent quantum-state manipulation of trapped atomic ions. J. Res. Natl. Inst. Stand. Tech. 103, 259 (1998).

[2] Ballance, C. J. et al. High-Fidelity Quantum Logic Gates Using Trapped-Ion Hyperfine Qubits. Phys. Rev. Lett. 117, 060504 (2016).

[3] Gaebler, J. P. et al. High-Fidelity Universal Gate Set for ${ }^{9} \mathrm{Be}^{+}$Ion Qubits. Phys. Rev. Lett. 117, 060505 (2016).

[4] Hempel, C. et al. Quantum Chemistry Calculations on a Trapped-Ion Quantum Simulator. Phys. Rev. X 8, 031022 (2018). 
[5] Anderegg, L. et al. Laser cooling of optically trapped molecules. Nature Physics 14, 890 (2018).

[6] Wilk, T. et al. Entanglement of Two Individual Neutral Atoms Using Rydberg Blockade. Phys. Rev. Lett. 104, 010502 (2010).

[7] Isenhower, L. et al. Demonstration of a Neutral Atom Controlled-NOT Quantum Gate. Phys. Rev. Lett. 104, 010503 (2010).

[8] Saffman, M. Quantum computing with atomic qubits and Rydberg interactions: progress and challenges. Journal of Physics B: Atomic, Molecular and Optical Physics 49, 202001 (2016).

[9] Wang, Y. et al. Single-qubit quantum memory exceeding ten-minute coherence time. Nature Photonics 11, 646 (2017).

[10] Chiaverini, J. et al. Realization of quantum error correction. Nature 432, 602 (2004).

[11] Schindler, P. et al. Experimental Repetitive Quantum Error Correction. Science 332, 1059 (2011).

[12] Wong-Campos, J. D. et al. Demonstration of Two-Atom Entanglement with Ultrafast Optical Pulses. Phys. Rev. Lett. 119, 230501 (2017).

[13] Schäfer, V. M. et al. Fast quantum logic gates with trapped-ion qubits. Nature 555, 75 (2018).

[14] Levine, H. et al. Parallel Implementation of High-Fidelity Multiqubit Gates with Neutral Atoms. Phys. Rev. Lett. 123, 170503 (2019).

[15] Graham, T. M. et al. Rydberg mediated entanglement in a two-dimensional neutral atom qubit array. arXiv 1908.06103 (2019).

[16] Zhang, S., Robicheaux, F. and Saffman, M. Magic-wavelength optical traps for Rydberg atoms. Phys. Rev. A 84, 043408 (2011).

[17] Savard, T. A., O'Hara, K. M. and Thomas, J. E. Laser-noise-induced heating in far-off resonance optical traps. Phys. Rev. A 56, R1095 (1997).

[18] Belyansky, R. et al. Nondestructive Cooling of an Atomic Quantum Register via StateInsensitive Rydberg Interactions. Phys. Rev. Lett. 123, 213603 (2019).

[19] Devoret, M. H. and Schoelkopf, R. J. Superconducting Circuits for Quantum Information: An Outlook. Science 339, 1169 (2013).

[20] Veldhorst, M. et al. A two-qubit logic gate in silicon. Nature 526, 410 (2015).

[21] Müller, M. et al. Trapped Rydberg ions: from spin chains to fast quantum gates. New Journal of Physics 10, 093009 (2008). 
[22] Feldker, T. et al. Rydberg Excitation of a Single Trapped Ion. Phys. Rev. Lett. 115, 173001 (2015).

[23] Higgins, G. et al. Single Strontium Rydberg Ion Confined in a Paul Trap. Phys. Rev. X 7, 021038 (2017).

[24] Higgins, G. et al. Coherent Control of a Single Trapped Rydberg Ion. Phys. Rev. Lett. 119, $220501(2017)$.

[25] Saffman, M., Walker, T. G. and Mølmer, K. Quantum information with Rydberg atoms. Rev. Mod. Phys. 82, 2313 (2010).

[26] Li, W. and Lesanovsky, I. Entangling quantum gate in trapped ions via Rydberg blockade. Appl. Phys. B 114, 37 (2014).

[27] Urban, E. et al. Observation of Rydberg blockade between two atoms. Nature Physics 5, 110 (2009).

[28] Gaëtan, A. et al. Observation of collective excitation of two individual atoms in the Rydberg blockade regime. Nature Physics 5, 115 (2009).

[29] Rao, D. D. B. and Mølmer, K. Robust Rydberg-interaction gates with adiabatic passage. Phys. Rev. A 89, 030301 (2014).

[30] Leibfried, D. et al. Creation of a six-atom Schrdinger cat state. Nature 438, 639 (2005).

[31] Higgins, G., Pokorny, F., Zhang, C. and Hennrich, M. Highly Polarizable Rydberg Ion in a Paul Trap. Phys. Rev. Lett. 123, 153602 (2019).

[32] Barrett, M. D. et al. Sympathetic cooling of ${ }^{9} \mathrm{Ba}^{+}$and ${ }^{24} \mathrm{Mg}^{+}$for quantum logic. Phys. Rev. A 68, 042302 (2003).

[33] Tan, T. R. et al. Multi-element logic gates for trapped-ion qubits. Nature 380, 528 (2015).

[34] Kielpinski, D., Monroe, C. and Wineland, D. J. Architecture for a large-scale ion-trap quantum computer. Nature 417, 709 (2002).

[35] Gambetta, F. M., Li, W., Schmidt-Kaler, F. and Lesanovsky, I. Engineering non-binary Rydberg interactions via electron-phonon coupling. arXiv 1907.11664 (2019).

[36] Wüster, S. and Rost, J.-M. Rydberg aggregates. J. Phys. B 51, 032001 (2018). 
Acknowledgements: We thank Klaus Mølmer for discussions and suggestions for the gate scheme. We thank all members of the ERyQSenS consortium for discussions. This work was supported by the European Research Council under the European Unions Seventh Framework Programme/ERC Grant Agreement No. 279508, the Swedish Research Council (Trapped Rydberg Ion Quantum Simulator), the QuantERA ERA-NET Cofund in Quantum Technologies (ERyQSenS), and the Knut \& Alice Wallenberg Foundation (Photonic Quantum Information). I.L. and W.L. acknowledge support from the EPSRC through Grant No. EP/M014266/1 and Grant No. EP/R04340X/1 via the QuantERA project ERyQSenS. I.L. also gratefully acknowledges funding through the Royal Society Wolfson Research Merit Award.

Author contributions: G.H., F.P. and M.H. built the experimental system, C.Z. and F.P. set up microwave dressing and improved the UV laser system, A.P. set up ablation loading of ions and the camera software, C.Z. had the idea to combine MW dressing and STIRAP excitation, C.Z. and G.H. carried out the measurements, C.Z. analysed the data, C.Z. and W.L. simulated the results, M.H. designed and administered the experiment, W.L. and I.L. calculated properties of atomic Rydberg states, W.L., I.L. and C.Z. analysed the scaling of the gate error. All authors contributed to discussion and writing the manuscript.

Data availability: The datasets generated during and analysed during the current study are available from the corresponding authors on reasonable request.

Competing interests: The authors declare that they have no competing financial interests.

Correspondence: Correspondence and requests for materials should be addressed to C.Z. (email: chi.zhang@fysik.su.se) or M.H. (email: markus.hennrich@fysik.su.se). 
TABLE I. Estimation and scaling of gate error from known sources. ${ }^{\text {a }}$

\begin{tabular}{c|ccc}
\hline Error source & This experiment $^{\mathrm{b}}$ & Scaling & Improved experiment $^{\mathrm{c}}$ \\
\hline Rydberg state decay & $3.5 \times 10^{-2}$ & $n^{-7} \cdot r^{3}$ & $5 \times 10^{-4}$ \\
Laser linewidth & $\approx 3 \times 10^{-2}$ & $\Gamma_{l} \cdot n^{-4} \cdot r^{3}$ & $2 \times 10^{-4}$ \\
Scattering via intermediate state $|e\rangle$ & $8 \times 10^{-3}$ & $\Delta^{-2} \cdot n^{4} \cdot r^{-3}$ & $8 \times 10^{-4}$ \\
Transitions within adiabatic basis & $5.5 \times 10^{-2}$ & $\left(\Omega_{\mathrm{max}}-\Delta / 2\right)^{-2} \cdot n^{4} \cdot r^{-3}$ & $4 \times 10^{-4}$ \\
MW power fluctuations ${ }^{\mathrm{d}}$ & $\approx 10^{-1}$ & $\delta \Omega_{\mathrm{MW}} \cdot n^{-4} \cdot r^{3}$ & $<10^{-5}$ \\
Coupling to dressed state $|-\rangle$ & $<10^{-4}$ & - & $<10^{-4}$ \\
Rydberg state polarisability & $3 \times 10^{-3}$ & - & $<10^{-4}$ \\
Coupling to motional modes & $\approx 10^{-4}$ & $N^{1 / 12}$ & $\approx 10^{-4}$ \\
Total error ${ }^{-4}$ & $\approx 23 \%$ & - & $0.19 \%$ \\
\hline
\end{tabular}

${ }^{a}$ More details about numerical simulation, derivation of the scaling, and estimation of the improved experimental parameters can be found in Supplemental Information.

b Two ions separated by $r=4.2 \mu \mathrm{m}$, sideband cooled to $\sim 98 \%$ population in motional ground state, excited to $n=46$ maximally-dressed Rydberg state with lifetime $\tau_{r} \simeq 7.8 \mu$ s (determined by experiment) and polarisability $\alpha \approx-6 \times 10^{-31} \mathrm{C}^{2} \mathrm{~m}^{2} \mathrm{~J}^{-1}$, interaction strength $V=V_{\max } \simeq 2 \pi \times 1.9 \mathrm{MHz}$. UV Rabi frequencies $\Omega_{1}=2 \pi \times 40 \mathrm{MHz}, \Omega_{2}=2 \pi \times 56.5 \mathrm{MHz}$, and UV laser linewidths $\Gamma_{1,2} \simeq 2 \pi \times 10 \mathrm{kHz}$.

${ }^{c}$ We consider a gate acting on two ions in a Doppler-cooled 100-ion crystal, the two ions are separated by $r=2.3 \mu \mathrm{m}$, and excited to the zero-polarisability Rydberg state with $n=60, \tau_{r} \simeq 25.5 \mu \mathrm{s}$ (natural lifetime), the residual polarisability $|\alpha|<10^{-34} \mathrm{C}^{2} \mathrm{~m}^{2} \mathrm{~J}^{-1}, V \simeq 2 \pi \times 21.9 \mathrm{MHz}, \Gamma_{1,2} \simeq 2 \pi \times 1 \mathrm{kHz}$, $\Omega_{1}=2 \pi \times 1000 \mathrm{MHz}$ and $\Omega_{2}=2 \pi \times 1414 \mathrm{MHz}$.

d The MW-dressed Rydberg state energy level depends on MW Rabi frequency (power), thus MW power fluctuations dephase the MW-dressed Rydberg state, causing infidelity.

${ }^{\text {e }}$ Coupling to the quadrupole field of the radio-frequency (rf) trap is not considered; the effect is negligible for $n=46$ state. For higher $n$, it may give a non-negligible energy shift oscillating with the quadrupole field and need to be compensated by modulating the laser and MW frequencies to achieve low error. 